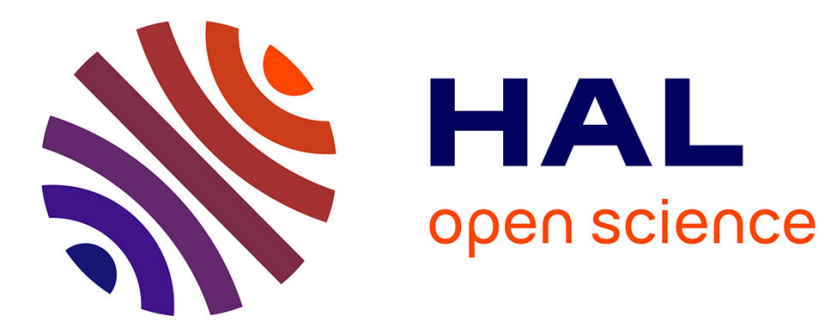

\title{
Surface waves in a magnetized ferrite slab filled with a wire medium
}

\author{
I.S. Nefedov, A.S. Soloviev, Anne-Claude Tarot, Wafa Abdouni
}

\section{To cite this version:}

I.S. Nefedov, A.S. Soloviev, Anne-Claude Tarot, Wafa Abdouni. Surface waves in a magnetized ferrite slab filled with a wire medium. European Physical Journal: Applied Physics, 2009, 46 (3), pp.1-5. 10.1051/epjap/2008194 . hal-00480140

\section{HAL Id: hal-00480140 https://hal.science/hal-00480140}

Submitted on 3 May 2010

HAL is a multi-disciplinary open access archive for the deposit and dissemination of scientific research documents, whether they are published or not. The documents may come from teaching and research institutions in France or abroad, or from public or private research centers.
L'archive ouverte pluridisciplinaire HAL, est destinée au dépôt et à la diffusion de documents scientifiques de niveau recherche, publiés ou non, émanant des établissements d'enseignement et de recherche français ou étrangers, des laboratoires publics ou privés. 


\title{
Surface waves in a magnetized ferrite slab filled with a wire medium
}

\author{
Igor S. Nefedov ${ }^{1}$, Andrey S. Soloviev ${ }^{2}$, Anne-Claude Tarot ${ }^{3}$, Wafa Abdouni ${ }^{3}$
}

September 5, 2008

\author{
${ }^{1}$ Department of Radio Science and Engineering, Helsinki University of Technology, P.O. \\ 3000, FI-02015 TKK, Finland. Fax:+358 9451 2152. Email: igor.nefedov@tkk.fi \\ ${ }^{2}$ Saratov State University, Astrakhanskaya 83, Saratov, Russia. \\ ${ }^{3}$ IETR, University de Rennes 1, Campus de Beaulieu, 35042 Rennes Cedex, France.
}

\begin{abstract}
Novel metamaterial, based on wire medium embedded into magnetized ferrite, is studied. Waves in unbounded ferrites filled with wire media, surface wave at the interface of this metamaterial and the air as well as waves in a ferrite slab adjacent to a wire medium are considered. Different geometries of wires arrangement and different magnetization directions are discussed. Effective permeability was introduced for the case where both plasma and magnetic properties take place. Dispersion diagrams and applicability of the Drude model for the description of the wire medium in a host matrix, possessing high permittivity and permeability, are discussed.
\end{abstract}

PACS. 41.20.-q Applied classical electromagnetism; 42.70.Qs Photonic bandgap materials; 75.30.Ds Spin waves

This paper belongs to the Special issue on Metamaterials, guest-editors: S. Zouhdi and A. Razek.

\section{Introduction}

In this paper we discuss electromagnetic properties of new metamaterial, based on the magnetized natural magnetics (ferrites) and the wire medium (WM) (the medium formed by a lattice of ideally conducting parallel wires, see $[1,2]$ ) If the wires are thin (compared with the lattice period), such the medium can be homogenized and its effective relative permittivity dyadic can be written as (wires are oriented in $z$ direction)

$$
\overline{\bar{\epsilon}}=\epsilon_{h}\left(\mathbf{u}_{x} \mathbf{u}_{x}+\mathbf{u}_{y} \mathbf{u}_{y}\right)+\epsilon_{z} \mathbf{u}_{z} \mathbf{u}_{z}
$$

where $\epsilon_{z}$ is expressed by the "plasma" (the Drude) formula

$$
\epsilon_{z}=\epsilon_{h}\left(1-\frac{\omega_{p}^{2}}{\omega^{2} \epsilon_{h} \mu_{h}}\right)=\epsilon_{h}\left(1-\frac{k_{p}^{2}}{k^{2} \epsilon_{h} \mu_{h}}\right) .
$$


Here $\epsilon_{h}$ and $\mu_{h}$ are permittivity and permeability of the host medium, respectively, $k=$ $\omega / c \sqrt{\epsilon_{h}}=k_{o} \sqrt{\epsilon_{h} \mu_{h}}, c$ is the speed of light, the constant $\omega_{p}$ (or corresponding $k_{p}$ ) is the equivalent "plasma frequency" for the case when the host medium is vacuum that gives grounds to call a wire medium as an "artificial plasma". (There exist different plasma models and we will use the formula for $k_{p}$ obtained in [3]). If the wavevector in WM has nonzero component along the wires, the plasma model Eq. 2 should be corrected introducing the spatial dispersion, see $[4,5]$.

It follows from Eq. 2, effective permittivity of WM can be made smaller than the permittivity of the host medium, even negative. Thus, embedding WM into ferrite, we can obtain material exhibiting high permeability and low effective dielectric constant. In next sections we will study wave properties of the ferrite - wire medium (FWM) as a PBG structure and examine the applicability of the effective medium theory for the description of FWM.

The paper is organized as follows: in Sec. 2 we present the effective medium model for waves propagating in unbounded transversally magnetized ferrite host matrix, filled with WM. In Sec. 3 the full-wave model, based on Green's function method, for the same structure is described. Dispersion diagram of FWM, considered as PBG structure, is discussed. Applicability of the effective medium model is demonstrated using results of Green's function method. In Sec. 4 surface waves in several structures containing ferrites and WM, whose geometries allow description in the framework of effective medium theory, are discussed.

\section{Effective medium model}

Let us consider a two-dimensional periodic (in $x$ and $y$ directions) lattice, formed of conductive, infinitely long wires, embedded into a magnetized YIG host matrix. Directions of the magnetic bias field $\mathbf{H}_{0}$ and the wave vector $\mathbf{k}$ are shown in Fig. 1. The relative permeability tensor takes form:

$$
\overline{\bar{\mu}}=\left(\begin{array}{lll}
\mu & -i \kappa & 0 \\
i \kappa & \mu & 0 \\
0 & 0 & 1
\end{array}\right)
$$

where

$$
\mu=1+\frac{\omega_{m}\left(\omega_{0}+i \alpha \omega\right)}{\left(\omega_{0}+i \alpha \omega\right)^{2}-\omega^{2}}, \quad \kappa=\frac{\omega_{m} \omega}{\left(\omega_{0}+i \alpha \omega\right)^{2}-\omega^{2}}
$$

$\omega_{m}=2 \pi \gamma M_{0}, \omega_{0}=2 \pi \gamma H_{0}, \alpha=\frac{2 \pi \gamma \Delta H}{2 \omega_{0}}$. Here $\gamma=2.8 \mathrm{GHz} / \mathrm{KOe}$ is the gyromagnetic ratio, $M_{0}$ is the saturation magnetization, $H_{0}$ is the biasing magnetic field and $\alpha$ is the damping factor, where $\Delta H$ is the ferromagnetic resonance linewidth. The value $\omega_{0}$ is referred to as the ferromagnetic resonant frequency. For an extraordinary wave, propagating transversely to $\mathbf{H}_{0}$ (in y direction for our case), the effective permeability can be introduced:

$$
\mu_{\mathrm{eff}}=\frac{\mu^{2}-\kappa^{2}}{\mu} .
$$

One can show that $\mu_{\mathrm{eff}} \rightarrow \infty$ if $\omega \rightarrow \omega_{\perp}=\sqrt{\omega_{0}\left(\omega_{0}+\omega_{m}\right)}$ in the lossless case $(\alpha=0)$, where $\omega_{\perp}$ is referred as the transversal ferromagnetic resonance frequency. High frequency magnetic 
field of this wave is parallel to $\mathbf{H}_{0}$. For an ordinary wave, having orthogonal polarization with the extraordinary one, effective $\mu$ is equal to static permeability which is unit in our case. Substituting $\mu_{\text {eff }}$ from (4) into Drude formula (2) one can find effective permittivity of FWM.

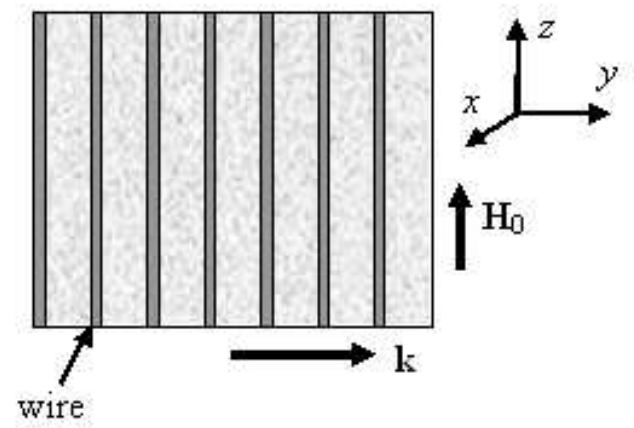

Figure 1: A wire medium embedded into ferrite host matrix.

\section{Numerical analysis and discussion}

First consider FWM as a PBG structure. Numerical analysis was implemented using Green's function in the spectral domain, derived in [6] and adopted to the 2D case, and Galerkin's method.

For the waves, propagating in the $x y$ plane we use the following representation of electric field, induced by current in wires:

$$
E_{z}(x, y)=\frac{\eta}{i k \epsilon_{h} \mu_{h}} \int_{L} G\left(x, y, x^{\prime}, y^{\prime}\right) J_{z}\left(x^{\prime}, y^{\prime}\right) d l^{\prime}
$$

where $\eta$ is the impedance of free space, $L$ is a contour of a perfectly conducting metal wire, $\mu_{h}$ is the effective permeability of the ferrite, given by formula (4) and the scalar Green function has the form

$$
\begin{aligned}
& G\left(x, y, z \mid x^{\prime}, y^{\prime}, z^{\prime}\right)= \\
& -\frac{1}{d^{2}} \sum_{m=-\infty}^{\infty} \sum_{n=-\infty}^{\infty} \frac{e^{-i\left[\alpha_{m}\left(x-x^{\prime}\right)+\beta_{n}\left(y-y^{\prime}\right)\right]}}{k^{2} \epsilon_{h} \mu_{h}-\alpha_{m}^{2}-\beta_{n}^{2}}
\end{aligned}
$$

where $\alpha_{m}=k_{x}+q_{m}, \beta_{n}=k_{y}+q_{n}, q_{s}=2 \pi s / d$. The values $\psi_{x}=k_{x} d$ and $\psi_{y}=k_{y} d$ have sense of phase shifts per lattice period $d$. Green's function (6) can be transformed to the form [6]:

$$
\begin{aligned}
& G(\bar{x}, \bar{y})=\frac{1}{2 d} \sum_{m=-\infty}^{\infty} \frac{e^{-i \alpha_{m} \bar{x}}}{t_{m}}\left[\frac{e^{t_{m}} \bar{y}_{e} e^{-i \psi_{y}-t_{m} d}}{1-e^{-\left(i \psi_{y}+t_{m} d\right)}}+\right. \\
& \left.\frac{e^{-t_{m} \bar{y}} e^{+i \psi_{y}-t_{m} d}}{1-e^{+i \psi_{y}-t_{m} d}}+e^{-t_{m}|\bar{y}|}\right],
\end{aligned}
$$

where $\bar{x}=x-x^{\prime}, \bar{y}=y-y^{\prime}, t_{m}=\sqrt{\alpha_{m}^{2}+\beta_{n}^{2}-k^{2} \epsilon_{h} \mu_{h}}$. The term

$$
G_{1}(\bar{x}, \bar{y})=\frac{1}{2 d} \sum_{m=-\infty}^{\infty} \frac{e^{-i \alpha_{m} \bar{x}} e^{-t_{m}|\bar{y}|}}{t_{m}}
$$

in (7) contains the logarithmic singularity. The series, asymptotic to (8), is

$$
\begin{aligned}
& \tilde{G}_{1}(\bar{x}, \bar{y})=\frac{e^{-i \alpha_{0} \bar{x}}}{2 \pi} \sum_{m=1}^{\infty} \frac{\cos m s}{m} e^{-m q}= \\
& =\frac{e^{-\alpha_{0} \bar{x}}}{4 \pi}\{q-\ln [2(\cosh q-\cos s)]\}
\end{aligned}
$$


where $s=\frac{2 \pi}{d} \bar{x}, q=\frac{2 \pi}{d} \overline{|y|}$ and

$$
\begin{gathered}
G_{1}(\bar{x}, \bar{y}) \rightarrow G_{0}(\bar{x}, \bar{y}) \equiv-\frac{e^{-\alpha_{0} \bar{x}}}{4 \pi} \ln \left[g^{2}\left(\bar{x}^{2}+\bar{y}^{2}\right)\right], \\
\text { when } \quad \bar{x} \rightarrow 0, \bar{y} \rightarrow 0 .
\end{gathered}
$$

Equating the left-hand side of (5) to zero at the surface of the conductor, one obtains the integral equation (IE):

$$
\int_{L} G\left(x, y, x^{\prime}, y^{\prime}\right) J_{z}\left(x^{\prime}, y^{\prime}\right) d l^{\prime}=0, \quad x \in L, y \in L .
$$

This first kind Fredholm's IE can be identically transformed to the second-kind IE:

$$
\begin{aligned}
& J(x, y) \int_{L} G_{0}\left(x, y \mid x^{\prime}, y^{\prime}\right) d x^{\prime} d y^{\prime}+ \\
& \int_{L}\left[G\left(x, y \mid x^{\prime}, y^{\prime}\right) J\left(x^{\prime}, y^{\prime}\right)-J(x, y) G_{0}\left(x, y \mid x^{\prime}, y^{\prime}\right)\right] d x^{\prime} d y^{\prime}=0, \\
& x \in L, y \in L
\end{aligned}
$$

The integrand in square brackets of (12) does not contain singularity, so this IE can be solved replacing the integral by a quadrature sum. Turning to the cylindrical coordinate system

$$
\begin{array}{ll}
x=r \cos \varphi & x^{\prime}=r^{\prime} \cos \varphi^{\prime} \\
y=r \sin \varphi & y^{\prime}=r^{\prime} \sin \varphi^{\prime},
\end{array}
$$

and using the rectangle summation rule, the contour integral can be expressed as

$$
\int_{0}^{2 \pi} f(r, \varphi) d \varphi \approx \frac{2 \pi}{N} \sum_{j=1}^{N-1} f\left(r \cos \varphi_{j}, r \sin \varphi_{j}\right)
$$

where $\varphi_{j}=2 \pi(j-1) / N$ and $N$ is the number of azimuth nodes. The contour integral over $G_{0}\left(x, y \mid x^{\prime}, y^{\prime}\right)$ can be evaluated analytically:

$$
I_{0}=\int_{0}^{2 \pi} G_{0}\left(r, \varphi \mid r, \varphi^{\prime}\right) d \varphi=-r \ln (r g)-\frac{r}{4} \ln 2 .
$$

Replacing integrals (12) by sums (14), one obtains the linear system of algebraic equations:

$$
B_{k} J_{k}+\sum_{j=1, j \neq k}^{N-1} A_{k j} J_{j}=0, \quad k=1, . . N-1,
$$

where unknowns are the values of the current at the nods $\varphi_{j}$,

$$
\begin{gathered}
A_{k j}=\frac{2 \pi}{N}\left[G\left(r, \varphi_{k} \mid r, \varphi_{j}\right)-\delta_{k j} G_{0}\left(r, \varphi_{k} \mid r, \varphi_{j}\right)\right], \\
B_{k}=I_{0} .
\end{gathered}
$$

Dispersion diagram for a square array of metal cylinders inside YIG is shown in Fig. 2. Following parameters are chosen: $M_{0}=1.75 \mathrm{KOe}, H_{0}=1.8 \mathrm{KOe}, \Delta_{H}=5 \mathrm{Oe}, \varepsilon_{h}=15$ (relative permittivity of YIG), period of WM lattice $d=5 \mathrm{~mm}$. In opposite to metal PBG structure with isotropic host matrix, dispersion of FWM structure is considerably determined by ferromagnetic resonant properties of the ferrite. It follows from Eq. 4, that there are two 
special frequency points, determining behavior of $\mu_{\text {eff }}$, namely, $f_{\perp}=\omega_{\perp} / 2 \pi=7.078 \mathrm{GHz}$ and $f_{b}=\left(\omega_{m}+\omega_{0}\right) / 2 \pi=9.94 \mathrm{GHz}$. At the frequency $f_{\perp} \mu_{\mathrm{eff}} \rightarrow \infty$ and $\mu_{\mathrm{eff}}=0$ if $f=f_{b}$.

Four pass bands are shown in Fig. 2. Grey area marks the frequency range, occupied by a countable spectrum of modes with the accumulation point $f_{\perp}$, where $\mu_{\text {eff }} \rightarrow \infty$. The stop band between $f_{\perp}$ and $f_{b}$ is caused by the negative value of $\mu_{\text {eff }}$. The value $\mu_{\text {eff }}$ is positive if the frequency satisfies $F>f_{b}$ but it grows slowly that causes large distances between pass bands in this frequency range.

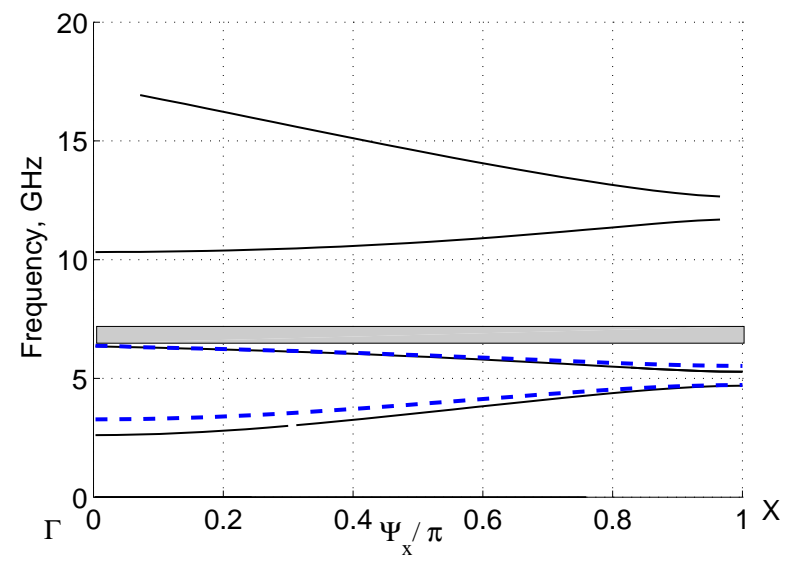

Figure 2: Dispersion diagram, calculated for $r=0.1 \mathrm{~mm}$ (solid curves) and $r=0.3 \mathrm{~mm}$ (dashed curves).

Figure 3 illustrates the applicability of the Drude formula for the description of FWM in the region of positive permittivity. Here $\varepsilon_{d}$ is the permittivity calculated using Eq. 4. More accurate $\varepsilon_{\text {eff }}$ is found from the numerical calculation of the propagation constant for the first-order pass band and is expressed as

$$
\varepsilon_{\mathrm{eff}}=\frac{\psi_{x}^{2}}{d^{2} \mu_{\mathrm{eff}}} .
$$

One can see that even for a small ratio $r / d=0.02$ the quite good agreement is observed only for small values $\varepsilon_{\text {eff }}$ and $\varepsilon_{d}$. For the ratio larger than $r / d=0.06$ the Drude formula can be used only for qualitative estimations of the effective permittivity. Note, that this consideration refers to the range of the small effective permeability $\mu_{e f f} \sim 2$.

\section{Surface waves at an interface air-FWM}

In this section we will consider several geometries which allow relatively simple description in the framework of effective medium theory.

\subsection{Geometry 1}

The first geometry is shown in Fig. 4. It follows from Maxwell's equations that for this geometry the waves can be split into ordinary wave with

$$
E_{x}=0, H_{y}=0, H_{z}=0, E_{y} \neq 0, E_{z} \neq 0, H_{x} \neq 0
$$




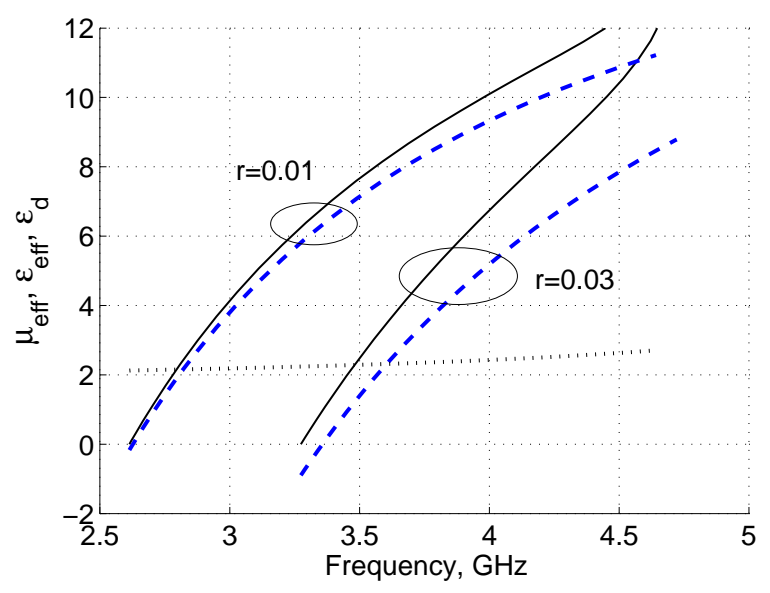

Figure 3: Effective $\mu$ (dotted curve), $\varepsilon_{\text {eff }}$, calculated using Eq. 19 (solid curves) and $\varepsilon_{d}$ (dashed curves).

and extraordinary wave with

$$
E_{x} \neq 0, H_{y} \neq 0, H_{z} \neq 0, E_{y}=E_{z}=H_{x}=0
$$

Fields of the ordinary wave do not interact with both wires and magnetic system. For the extraordinary wave the known dispersion equation for surface waves at the interface air-tangentially magnetized ferrite [7] can be used:

$$
\mu \mu_{\perp} \beta \pm \gamma \mu_{a}+\alpha \mu=0
$$

where $\gamma$ is the propagation constant of the surface wave, $\alpha=\sqrt{\gamma^{2}-k^{2} \epsilon_{\text {eff }} \mu_{\perp}}, \beta=\sqrt{\gamma^{2}-k^{2}}$,

$$
\epsilon_{\mathrm{eff}}=\epsilon_{x x}=\epsilon_{f}\left(1-\frac{k_{p}^{2}}{k^{2} \epsilon_{f} \mu_{\perp}}\right) .
$$

Thus WM in this geometry can be described in the framework of the effective medium theory where the spatial dispersion does not take place. Dispersion diagram is shown in Fig. 5. Propagation of the surface wave demonstrates the non-reciprocity which is caused by a shift of the maximum of the high-frequency field in the direction, orthogonal to the magnetization. There is the analogy with waves in a tangentially magnetized ferrite slab, located between air on one side and metal on the other side. The surface wave, propagating along the interface air-ferrite has the limiting frequency point $f_{\text {air }}=F_{0}+F_{m} / 2$ and the wave propagating at the metal interface has $f_{\text {met }}=F_{0}+F_{m}$. Dispersion curve calculated at $H_{0}=2.9$ kOe illustrates the tunability of the structure.

\subsection{Geometry 2. Inverted structure}

In this subsection the wave propagation in an inverted structure which is a ferrite adjacent to WM (see Fig. 6), is discussed. Here we observe that the reduction of thickness of the ferrite slab shifts up dispersion curves. In opposite to the previous case, the dispersion is negative at small $\gamma$. Note, areas with negative dispersion are usual in finite-thickness ferrite films [8]. The 


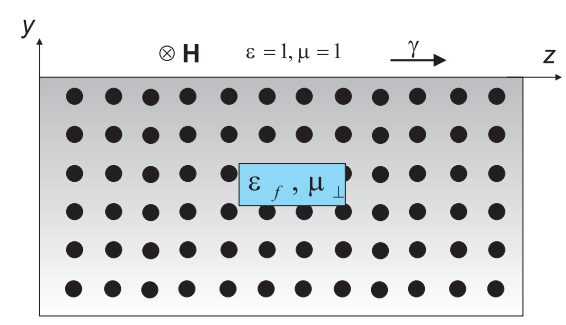

Figure 4: Geometry 1. Interface air - wire medium, inserted into the ferrite, magnetized along the wires.

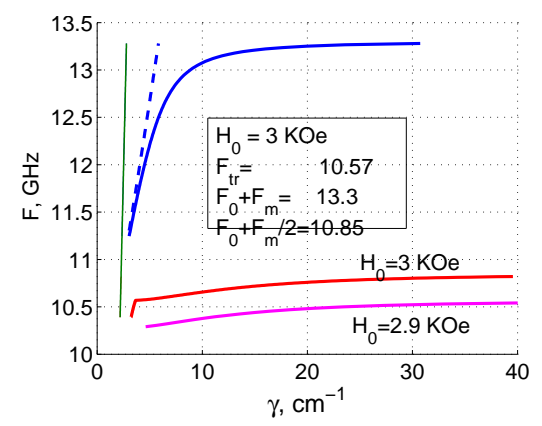

Figure 5: Dispersion of surface waves propagating in opposite directions along the interface.

curves 1,2 and 3 correspond to the wave, whose field is localized at the interface ferrite-WM. In the limit $\gamma \rightarrow \infty$ the cutoff frequency tends to $f_{\text {air }}$, however the effective medium model fully fails at large wavenumbers $\gamma$. For the wave, propagating in the opposite direction, whose field is localized at the metal, we have shown the low- $\gamma$ part of the dispersion curve. At larger $\gamma$ the dispersion is the same as for the surface wave propagating along the metal boundary of a semi-infinite ferrite.

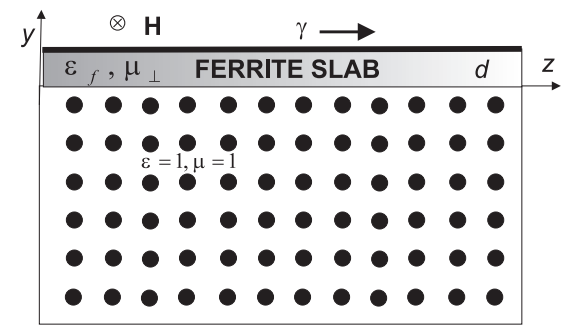

Figure 6: Geometry 2. Ferrite slab adjacent to the metal plane (on top) and the WM in air matrix (on the bottom)

\subsection{Geometry 3}

Let us consider the next geometry, see Fig. 8. Maxwell's equations for the fields in this structure are split also into two subsystems, corresponding to the TE wave with

$$
E_{x} \neq 0, H_{y} \neq 0, H_{z} \neq 0 ; E_{y}=E_{z}=H_{x}=0
$$




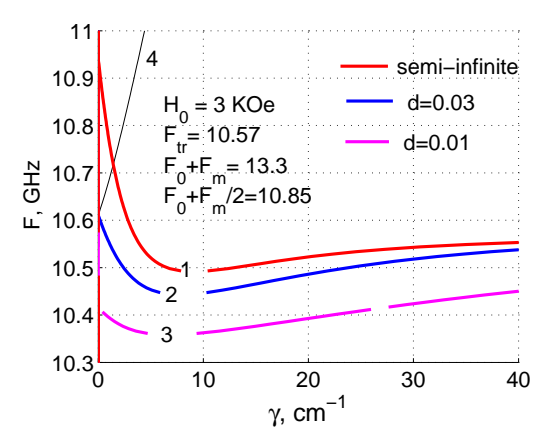

Figure 7: Dispersion diagram for surface waves propagating at the interface ferrite-WM. The curve number 1 corresponds to the semi-infinite ferrite, the curve 2 corresponds to the slab with the thickness $d=0.3 \mathrm{~cm}$, the curve $3-d=0.01 \mathrm{~cm}$. The curve 4 is the low-wavenumber part of the dispersion diagram for the surface wave propagating in the opposite direction.

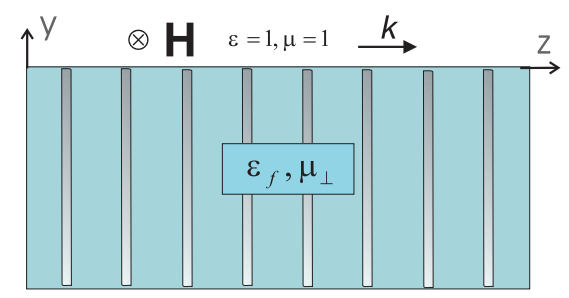

Figure 8: Geometry 3. Wires are normal to the interface.

and the TM+TEM waves with

$$
H_{x} \neq 0, E_{y} \neq 0, E_{z} \neq 0 ; H_{y}=H_{z}=E_{x}=0 .
$$

The TE wave is usual surface Damon-Eshbach wave [9] and wires do not affect this wave. Regarding the other polarization, propagation of two waves, TM and TEM is possible [5]. They are coupled at the interface and additional boundary conditions are necessary for the solution of boundary-value problems [10]. There are no purely surface waves because TEM mode will carry away energy from the interface and it is reasonable only to consider waves in a finite-thickness slab.

\subsection{Geometry 4}

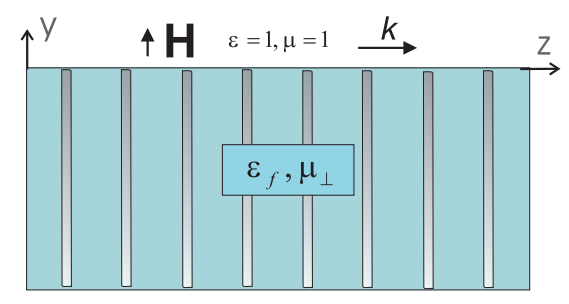

Figure 9: Geometry 4.

Waves in this structure, see Fig. 9, are not split into subsystems and all field components are present. Homogenization is needed. 


\section{Conclusion}

Novel metamaterial, composed of the magnetized ferrite and wire medium, is described. It can be used as a low-loss tunable metamaterial in microwave range. Analysis made for an unbounded FWM is valid for a finite thickness slab placed between two metal planes (patch and ground plane) if the field does not depend on the $z$ coordinate. It was demonstrated, that for high values of the permittivity of the host medium the Drude formula gives essential error for the calculation of $\epsilon_{\text {eff }}$.

\section{Acknowledgment}

The authors would like to thank the CNRS (Centre National de la Recherche Scientifique) for its financial support.

\section{References}

[1] J. Brown, Progress in Dielectrics, 2, 195 (1960).

[2] J.B. Pendry, A.J. Holden, W.J. Stewart, I. Youngs, Phys. Rev. Lett. 76, 4773 (1996).

[3] P.A. Belov, S.A. Tretyakov, A.J. Viitanen, J. of Electrom. Waves and Applic. 16, 1153 (2002).

[4] G. Shvets, in Advanced Accelerator Concept: Tenth Workshop, edited by C.E. Clayton and P. Muggli (American Institute of Physics, 2002), p. 371.

[5] P.A. Belov, R. Marques, S.I. Maslovski, I.S. Nefedov, M. Silveirinha, C.R. Simovski, S.A. Tretyakov, Phys. Rev. B 63, 113103 (2003).

[6] I.S. Nefedov, A.J. Viitanen, S.A. Tretyakov, Phys. Rev. E, 71, 046612 (2005).

[7] T.J. Gerson, J.S. Nadan, IEEE Trans. on Micr. Theory Tech. MTT-22 707 (1974).

[8] V.N. Ivanov, N.P. Demchenko, I.S. Nefedov, R.A. Silin, A.G. Schuchinsky, Izvestiya Vysshikh Uchebnykh Zavedenii. Seriya Radiofizika 32, 764 (1989) (in Russian). (English translation: Radiophysics and Quantum Electronics, 32577 (1989).

[9] J.R. Eshbach, R.W. Damon, Phys. Rev. 118, 1208 (1960).

[10] M. Silveirinha, IEEE Trans. on Ant. and Prop. 54, 1766 (2006). 